\title{
Adult-onset diabetes
}

National Diabetes Information Clearinghouse (NDIC)

\section{Definitions}

Type 2 diabetes

Defined by National Diabetes Information Clearinghouse (NDIC)

\section{Source}

National Diabetes Information Clearinghouse (U.S.). (2009). The diabetes dictionary. [Bethesda, Md.]: U.S. Dept. of Health and Human Services, National Institutes of Health, National Institute of Diabetes and Digestive and Kidney Diseases, National Diabetes Information Clearinghouse.

Former term for type 2 diabetes. 